\title{
NMR Characterization of Oxidized Form of Human 8-kDa Dynein Light Chain
}

\author{
Jae-Sun Shin ${ }^{1}$, Woojin Jeong ${ }^{2, *}$, and Seung-Wook Chi ${ }^{1, *}$ \\ ${ }^{1}$ Medical Proteomics Research Center, KRIBB, Daejeon 305-806, Korea, \\ ${ }^{2}$ Department of Life Science, Division of Life and Pharmaceutical Sciences, \\ Ewha Womans University, Seoul 120-750, Korea \\ (Received Oct 30, 2010; accepted Dec 9, 2010)
}

\begin{abstract}
Redox-dependent conformational change of human 8-kDa Dynein light chain (LC8) plays important role in regulating NF- $\mathrm{KB}$ signaling pathway. In this study we characterized the structural states of the oxidized and reduced forms of LC8 by using NMR spectroscopy. The ${ }^{1} \mathrm{H}^{-15} \mathrm{~N} 2 \mathrm{D}$ HSQC spectra of oxidized LC8 indicated that no significant change in tertiary structure of LC8 occurred upon oxidation. The chemical shift perturbations of LC8 upon oxidation suggest a redox-dependent quaternary structural change.
\end{abstract}

Keywords : LC8, NMR, Redox change; Dynein

\section{INTRODUCTION}

Dyneins are multi-subunit complexes that deliver molecular cargoes via microtubules. Human 8-

kDa dynein light chain (LC8) is a main component of dynein motor complex. ${ }^{1}$ In Drosophilia, loss of

LC8 function induces severe defects in development of bristle, wing and sensory axon projections.

LC8 plays main dual role in maintaining structural integrity of protein complex and exerting cellular response against various signaling stimuli such as tumor necrosis factor- $\alpha$ (TNF- $\alpha$ ) signaling and

\footnotetext{
* To whom correspondence should be addressed. E-mail : swchi@kribb.re.kr; jeongw@ewha.ac.kr
} 
redox change. LC8 regulates various biological processes via interaction with a number of binding

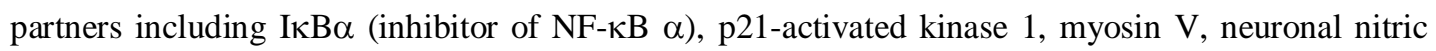
oxide synthase (nNOS), and the pro-apoptotic Bcl-2 family proteins Bmf and Bim. ${ }^{2}$

Recently, it was reported that the reduced LC8 directly interacts with IкB $\alpha$ and the interaction is associated with NF-kB signaling in response to cellular redox change. ${ }^{3}$ The interaction of LC8 with

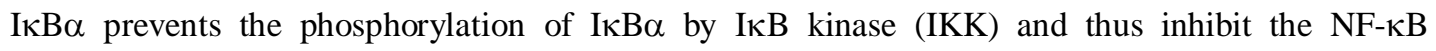
signaling pathway. Upon sensing TNF- $\alpha$-induced reactive oxygen species (ROSs), LC8 is oxidized via the disulfide bond formation between two Cys2 of each subunit and the homodimer is then

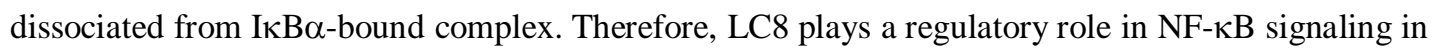
response to cellular redox change and thus redox state-dependent conformational change of LC8 is important for its regulatory function.

Although the functional activity of LC8 is highly coupled to redox change, its correlation with structural change remains still unclear. All the structures of LC8 solved until now are restricted to reduced forms. Structure of reduced form of rat LC8 at $\mathrm{pH} 7$ was dimeric ${ }^{2}$ whereas that of Drosophilia LC8 at pH 3 was monomeric. ${ }^{4}$ Characterization of oxidized LC8 structure is necessary to elucidate the molecular basis for the redox state-dependent conformational change. In this study, we have characterized the structural status of oxidized form of LC8 by using NMR spectroscopy.

\section{EXPERIMENTAL}




\section{Protein purification}

A DNA fragment encoding human LC8 was cloned into the NdeI and BamH1 sites of pET15b vector and $\mathrm{N}$-terminal $\mathrm{His}_{6}$-tagged recombinant protein was expressed in Escherichia coli BL21(DE3). The ${ }^{15} \mathrm{~N}$-labeled LC8 was purified by Nickel affinity column as previously described. ${ }^{5-6}$

\section{NMR Spectroscopy}

All the NMR spectra were obtained at $30{ }^{\circ} \mathrm{C}$ using a Bruker Avance II 900 spectrometers equipped with cryogenic probes. The ${ }^{1} \mathrm{H}^{-15} \mathrm{~N}$ 2D HSQC spectra for oxidized and reduced forms of human LC8 were acquired. The NMR samples containing $160 \mu \mathrm{M}^{15} \mathrm{~N}$-labeled LC8 were prepared in $90 \% \mathrm{H}_{2} \mathrm{O} / 10 \%{ }^{2} \mathrm{H}_{2} \mathrm{O}, 20 \mathrm{mM}$ TrisHCl (pH 7.5) for oxidized form and 1 mM EDTA and 5 mM DTT were added for reduced form. All data were processed and analyzed using an nmrPipe/nmrDraw and SPARKY software.

\section{RESULTS AND DISCUSSION}

Although the functional activity of LC8 was shown to depend on cellular redox change, its structures determined to date have been confined to the reduced forms. The previously reported functional switch of $\mathrm{LC}^{3}$ suggests the redox state-dependent conformational change. Recently, it was shown that Cys2 of LC8 may be involved in intermolecular disulfide bond formation. The two 
Cys2 in the previously determined structures of reduced LC8 are located at the opposed faces of the dimer (Fig. 1). The two Cys2 are thus far from each other, separated by 54 $\AA$. Based on the structure of reduced LC8, we postulate that fairly large conformational change should occur upon intermolecular disulfide bond formation by oxidation.

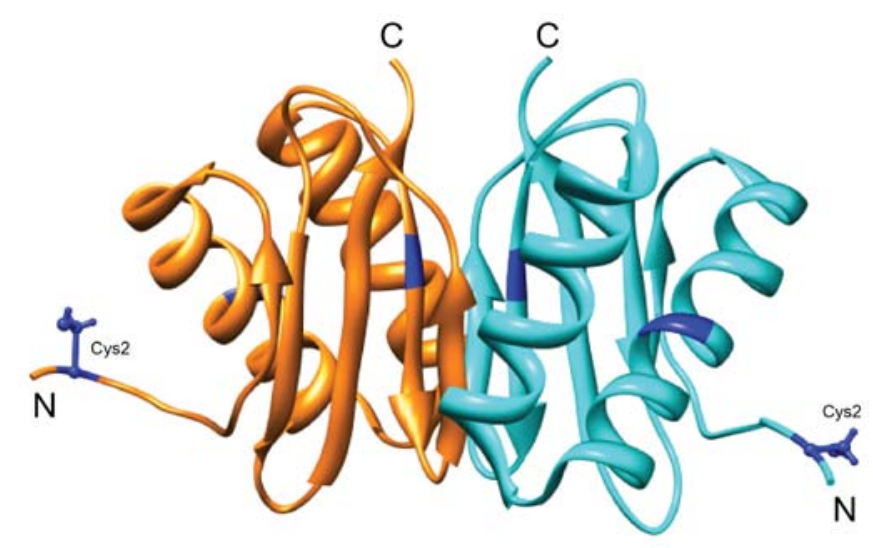

Figure. 1. Dimeric structure of reduced LC8 (PDB code: 1F3C). Cysteine residues were colored in blue and Cys2 residues were shown in ball and stick.

To test this hypothesis, we expressed and purified the oxidized and reduced forms of human LC8 and acquired the ${ }^{1} \mathrm{H}^{-15} \mathrm{~N}$ 2D HSQC spectra of them. Figures 2 A and $2 \mathrm{~B}$ shows ${ }^{1} \mathrm{H}-{ }^{15} \mathrm{~N} 2 \mathrm{D}$ HSQC spectra for the oxidized and reduced forms of human LC8, respectively. As shown in the overlaid HSQC spectra for both of them (Fig. 2 C), the crosspeaks of oxidized form of human LC8 are similar to those of reduced form except that several crosspeaks from flexible N-terminal residues. 
In particular, no chemical shift perturbation was observed on Cys2 of LC8 (Fig. 2 C). This indicates that no significant change in tertiary structure of human LC8 occurred upon oxidation.

Resonance assignments for ${ }^{15} \mathrm{~N}-{ }^{1} \mathrm{H}$ HSQC spectra of the oxidized LC8 protein were preformed on the basis of the previously reported assignment of reduced LC8. ${ }^{2}$ The significant chemical shift perturbations of LC8 upon oxidation were observed on residues 1, 3, 5, 6, 14, 33, 34, 35, 58, 70, 81, 82 and 87. Except the N-terminal flexible residues, the perturbations were mapped onto the reduced dimeric structure of LC8 (Fig. 3). The significant chemical shift perturbation of LC8 crosspeaks may arise from intersubunit contacts of LC8 via red colored residues (Fig. 3), which suggest the possibility that quaternary structural change resulted from oxidation process. Oxidized LC8 seems to be tetrameric or higher-order oligomeric whereas reduced LC8 was reported to be dimeric or monomeric. Therefore, not tertiary structural change but quaternary structural change may lead to redox-dependent conformational change of LC8.

Change of quaternary structure or oligomerization is a highly conserved mechanism to regulate the function of redox-sensing proteins such as molecular chaperones. Although the oligomeric status varies depending on the protein, it can provide an effective biological mechanism to change the binding site of partner proteins. This is reminiscent of the functional switch mechanism of Heat shock protein 33 (Hsp33) by oligomerization, where oxidized dimer or higher-order oligomer is active molecular chaperone but reduced monomeric form is inactive. ${ }^{7-8}$ 

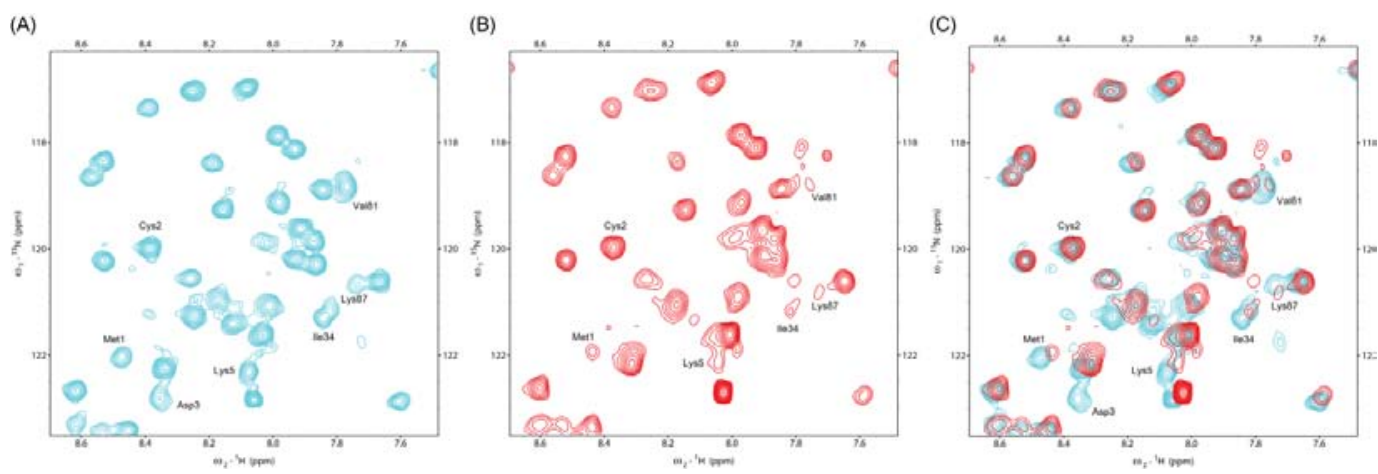

Figure. $2 .{ }^{1} \mathrm{H}^{15} \mathrm{~N}$ 2D HSQC spectra of reduced LC8 (A) and oxidized LC8 (B). (C) The overlaid spectra for reduced (cyan) and oxidized LC8 (red). The LC8 residues that were changed upon oxidation were labeled.

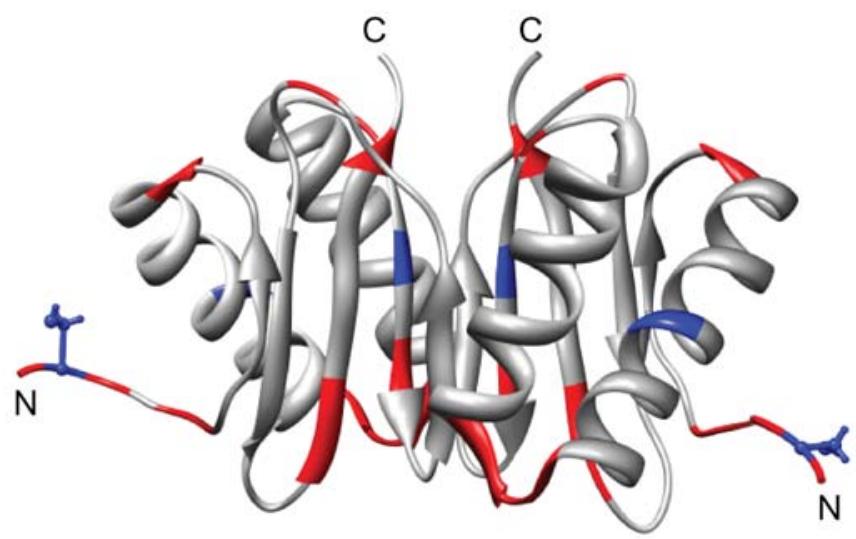

Figure. 3. Chemical shift perturbation in reduced LC8 upon oxidation. Cysteine residues are colored in blue and residues that show significant chemical shift perturbation upon oxidization are colored in red.

To gain insight into a molecular basis for the conformational change of human LC8 upon redox change, we characterized the structural states of oxidized and reduced forms of the LC8 using NMR 
spectroscopy. Our results indicated that there is no significant change in the tertiary structure of LC8 upon oxidation. The observed chemical shift perturbations of LC8 upon oxidation suggest change of quaternary structure upon redox change.

\section{Acknowledgment}

This work was supported by Mid-career Researcher Program through NRF grant funded by the MEST (No. 2008-0061624). This study made use of the NMR facility at Korea Basic Science Institute, which is supported by Advanced Bio-MR technology Program of the Korean Ministry of Education, Science and Technology (T29220).

\section{REFERENCES}

1. S.M. King, Biochim Biophys Acta 1496, 60. (2000).

2. J. Fan, Q. Zhang, H. Tochio, M. Li, M. Zhang, J. Mol. Biol. 306, 97. (2001).

3. Y. Jung, H. Kim, S.H. Min, S.G. Rhee, W. Jeong, J. Biol. Chem. 283, 23863. (2008).

4. M. Makokha, Y.J. Huang, G. Montelione, A.S. Edison, E. Barbar, Protein Sci. 13, 727. (2004).

5. J.-H. Kim, K.-Y. Lee, S.-J. Park, nd B.-J. Lee, J. Kor. Magn. Reson. Soc. 14, 45. (2010).

6. W. Koh, M. Kim, S. Moon, W. Lee, J. Kor. Magn. Reson. Soc. 13, 56. (2009).

7. M.W. Akhtar, V. Srinivas, B. Raman, T. Ramakrishna, T. Inobe, K. Maki, M. Arai, K. Kuwajima, C.M. Rao, J. Biol. Chem. 279, 55760. (2004).

8. S.J. Kim, D.G. Jeong, S.-W. Chi, J.S. Lee, S.E. Ryu, Nat. Struct. Biol. 8, 459. (2001). 